\title{
The effect of points fattening in dimension three
}

\author{
Th. Bauer ${ }^{1}$ and T. Szemberg ${ }^{2}$
}

January 12, 2014

\begin{abstract}
There has been increased recent interest in understanding the relationship between the symbolic powers of an ideal and the geometric properties of the corresponding variety. While a number of results are available for the twodimensional case, the higher-dimensional case is largely unexplored. In the present paper we study a natural conjecture arising from a result by Bocci and Chiantini. As a first step towards understanding the higher-dimensional picture, we show that this conjecture is true in dimension three. Also, we provide examples showing that the hypotheses of the conjecture may not be weakened.
\end{abstract}

\section{Dedication}

This article is dedicated to Robert Lazarsfeld on the occasion of his 60th birthday.

\section{Introduction}

The study of the effect of points fattening has been initiated by Bocci and Chiantini in [3]. Roughly speaking, they consider the radical ideal $I$ of a finite set $Z$ of points in the projective plane, its second symbolic power $I^{(2)}$, and deduce from the comparison of algebraic invariants of these two ideals various geometric properties of the set $Z$. Along these lines Dumnicki, Tutaj-Gasińska and the second author studied in [7] higher symbolic powers of $I$. Similar problems were studied in [1] in the bi-homogeneous setting of ideals defining finite sets of points in $\mathbb{P}^{1} \times \mathbb{P}^{1}$.

It is a natural task to try to generalize the result of Bocci and Chiantini [3, Theorem 1.1] to the higher-dimensional setting. Denoting for a homogeneous ideal $I$ by $\alpha(I)$ its initial degree, i.e., the least degree $k$ such that $(I)_{k} \neq 0$, a natural generalization reads as follows:

Conjecture 1.1. Let $Z$ be a finite set of points in projective space $\mathbb{P}^{n}$ and let $I$ be the radical ideal defining $Z$. If

$$
d:=\alpha\left(I^{(n)}\right)=\alpha(I)+n-1,
$$

then either

\footnotetext{
${ }^{1}$ Partially supported by DFG grant BA $1559 / 6-1$

${ }^{2}$ Partially supported by NCN grant UMO-2011/01/B/ST1/04875
} 
$\alpha(I)=1$, i.e., $Z$ is contained in a single hyperplane $H$ in $\mathbb{P}^{n}$

or

$Z$ consists of all intersection points (i.e., points where $n$ hyperplanes meet) of a general configuration of $d$ hyperplanes in $\mathbb{P}^{n}$, i.e., $Z$ is a star configuration. For any polynomial in $I^{(n)}$ of degree $d$, the corresponding hypersurface decomposes into d such hyperplanes.

The term general configuration in the conjecture means simply that no more than $n$ hyperplanes meet in one point. This is equivalent to general linear position for points in the dual projective space corresponding to the hyperplanes in the configuration. The result of Bocci and Chiantini is the case $n=2$ of this conjecture. As a first step towards understanding the higher-dimensional picture, we show in the present paper:

Theorem 1.2. The conjecture is true for $n=3$.

The assumption on the ideal $I$ in the theorem amounts to the two equalities

$$
\begin{aligned}
& \alpha\left(I^{(2)}\right)=\alpha(I)+1 \\
& \alpha\left(I^{(3)}\right)=\alpha\left(I^{(2)}\right)+1
\end{aligned}
$$

and one might be tempted to relax the assumptions to only one of them. In Sect. 6 we provide examples showing, however, that neither is sufficient by itself to get the conclusion of the theorem.

Star configurations are interesting objects of study in their own right. They are defined in [10] as unions of linear subspaces of fixed codimension $c$ in projective space $\mathbb{P}^{n}$ that result as subspaces where exactly $c$ of a fixed finite set of general hyperplanes in $\mathbb{P}^{n}$ intersect. The case described in Conjecture 1.1 corresponds thus to the $c=n$ situation. It is natural to wonder if the following further generalization of Conjecture 1.1 might be true: If $Z$ is a finite collection of linear subspaces of codimension $c \leqslant n$ in $\mathbb{P}^{n}$ with the radical ideal $I$ and such that

$$
d=\alpha\left(I^{(c)}\right)=\alpha(I)+c-1,
$$

then $Z$ is either contained in a hyperplane or forms a star configuration of codimension $c$ subspaces. The recent preprint [12] of Janssen deals with lines in $\mathbb{P}^{3}$ and shows that such a simple generalization would be too naive. Nevertheless we expect that there are some undiscovered patterns lurking behind, and we hope to come back to this subject in the near future.

Throughout the paper we work over the complex numbers, and we use standard notation in algebraic geometry as in [13.

\section{Initial degrees of symbolic powers}

Definition 2.1 (Symbolic power). Let $I$ be a homogeneous ideal in the polynomial ring $R=\mathbb{C}\left[\mathbb{P}^{n}\right]$. For a positive integer $k$, the ideal

$$
I^{(k)}=R \cap\left(\bigcap_{\mathfrak{p} \in \operatorname{Ass}(I)} I^{k} R_{\mathfrak{p}}\right),
$$


where the intersection is taken in the field of fractions of $R$, is the $k$-th symbolic power of $I$.

Definition 2.2 (Differential power). Let $I$ be a radical homogeneous ideal and let $V \subset \mathbb{P}^{n}$ be the corresponding subvariety. For a positive integer $k$, the ideal

$$
I^{\langle k\rangle}=\bigcap_{P \in V} \mathfrak{m}_{P}^{k},
$$

where $\mathfrak{m}_{P}$ denotes the maximal ideal defining the point $P \in \mathbb{P}^{n}$, is the $k$-th differential power of $I$.

In other words, the $k$-th differential power of an ideal consists of all homogeneous polynomials vanishing to order at least $k$ along the underlying variety. For radical ideals these two concepts fall together due to a result of Nagata and Zariski, see [8, Theorem 3.14] for prime ideals and [14, Corollary 2.9] for radical ideals:

Theorem 2.3 (Nagata, Zariski). If I is a radical ideal in a polynomial ring over an algebraically closed field, then

$$
I^{\langle k\rangle}=I^{(k)}
$$

for all $k \geqslant 1$.

We will make use of the following observation on symbolic powers.

Lemma 2.4. Let I be an arbitrary radical homogeneous ideal. Then we have the inequality

$$
\alpha\left(I^{(k+1)}\right)-\alpha\left(I^{(k)}\right) \geqslant 1
$$

for all $k \geqslant 1$.

Proof. Let $Z$ be the subscheme of $\mathbb{P}^{n}$ defined by $I$. The claim of the lemma follows immediately from the interpretation of symbolic powers as differential powers, Theorem 2.3. and the observation that if a polynomial $f$ vanishes along $Z$ to order at least $k+1$, then any of its partial derivatives vanishes along $Z$ to order at least $k$.

\section{$3 \quad$ The $\mathbb{P}^{2}$ case revisited}

As a warm-up, we give here a new proof of the result of Bocci and Chiantini. This proof has the advantage that it does not make use of the Plücker formulas.

Theorem 3.1 (Bocci-Chiantini). Let $Z$ be a finite set of points in the projective plane $\mathbb{P}^{2}$ and let $I$ be its radical ideal. If

$$
d=\alpha\left(I^{(2)}\right)=\alpha(I)+1,
$$

then either $Z$ consists of collinear points or $Z$ is the set of all intersection points of a general configuration of $d$ lines in $\mathbb{P}^{2}$. 
Proof. If $d=2$, then we are done. So we assume $d \geqslant 3$.

By Lemma 4.2 below we may assume that $Z$ consists of exactly $\left(\begin{array}{l}d \\ 2\end{array}\right)$ points. Let $X_{2}$ be a divisor of degree $d$ that is singular in all points of $Z$. Let $P$ be one of the points in $Z$. Then there exists a divisor $W_{P}$ of degree $d-2$ vanishing at all points in $Z \backslash\{P\}$ (and not vanishing at $P$ ).

We claim that $W_{P}$ is contained in $X_{2}$. To see this, we begin by showing that they must have a common component. Indeed, this follows from Bézout's Theorem, since otherwise we would get

$$
d(d-2)=X_{2} \cdot W_{P} \geqslant 2\left(\left(\begin{array}{l}
d \\
2
\end{array}\right)-1\right)=d(d-1)-2,
$$

which is equivalent to $d \leqslant 2$ and contradicts our initial assumption in this proof.

Let now $\Gamma$ be the greatest common divisor of $X_{2}$ and $W_{P}$, and let $e$ be the degree of the divisor $W_{P}^{\prime}=W_{P}-\Gamma$ (so that $\operatorname{deg}(\Gamma)=d-2-e$ ). There must be at least $\left(\begin{array}{c}e+2 \\ 2\end{array}\right)-1$ points from $Z \backslash\{P\}$ on $W_{P}^{\prime}$ (otherwise there would be a pencil of such divisors $W_{P}^{\prime}$ and one could choose an element in this pencil passing through $P$, but then $W_{P}^{\prime}+\Gamma$ would be an element of degree $d-2$ in $I$ contradicting the assumption on $\alpha(I))$.

Then we intersect again $X_{2}-\Gamma$ with $W_{P}^{\prime}$ and obtain

$$
(e+2) e=\left(X_{2}-\Gamma\right) \cdot W_{P}^{\prime} \geqslant 2\left(\left(\begin{array}{c}
e+2 \\
2
\end{array}\right)-1\right)=(e+2)(e+1)-2,
$$

which gives $e=0$.

It follows that $X_{2}-W_{P}$ is a divisor of degree 2 with a double point at $P$. Hence $X_{2}$ contains two lines intersecting in $P$. This holds for every point $P \in Z$. Since $X_{2}$ can contain at most $d$ lines, we see that this is only possible if $Z$ consists of the intersection points of a general configuration of $d$ lines.

\section{A reduction result}

We begin by a lemma concerning star configurations of points. We include the proof, since we were not able to trace it down in the literature.

Lemma 4.1. Let $Z$ be a star configuration of points defined by hyperplanes $H_{1}, \ldots, H_{d}$ in $\mathbb{P}^{n}$. For $d \geqslant n+1$ the union

$$
H_{1} \cup \ldots \cup H_{d}
$$

is the only hypersurface $F$ of degree d with the property

$$
\operatorname{mult}_{P} F \geqslant n \text { for all } P \in Z \text {. }
$$

Proof. We proceed by induction on the dimension $n \geqslant 2$. The initial case of $\mathbb{P}^{2}$ is simply dealt with by a Bézout type argument. Indeed, assuming that there exists a curve $F$ of degree $d$ passing through all points in $Z$ with multiplicity $\geqslant 2$ and taking a configuration line $H_{i}$, Bézout's theorem implies that $H_{i}$ is a component of $F$. Since this holds for all lines in the configuration and $\operatorname{deg}(F)=d$, we are done. 
For the induction step we assume that the lemma holds for dimension $n-1$ and all $d \geqslant n$. We want to conclude that it holds for $\mathbb{P}^{n}$ and all $d \geqslant n+1$. Of course we may assume that $n \geqslant 3$.

To this end let $F$ be a hypersurface of degree $d$ in $\mathbb{P}^{n}$ satisfying (2). Suppose that there exists a hyperplane $H$ among $H_{1}, \ldots, H_{d}$, which is not a component of $F$. Then the restriction $G=F \cap H$ is a hypersurface of degree $d$ in $H \simeq \mathbb{P}^{n-1}$ with mult $_{P} G \geqslant n$ for all $P \in Z_{H}=Z \cap H$. Note that $Z_{H}$ is itself a star configuration of points in $H$, defined by hyperplanes obtained as intersections $H_{i} \cap H$. So it is a star configuration of $d-1$ hyperplanes in $\mathbb{P}^{n-1}$. The polar system of $G$ (i.e., the linear system defined by all first order derivatives of the equation of $G$ ) is of dimension $n-1 \geqslant 2$, and every element $K$ in this system satisfies

$$
\operatorname{mult}_{P} K \geqslant n-1 \quad \text { for all } P \in Z_{H} .
$$

This contradicts the induction assumption.

The following lemma allows one to assume $\# Z=\left(\begin{array}{l}d \\ n\end{array}\right)$ when proving the theorem (or when working on the conjecture).

Lemma 4.2. Suppose that the set $Z \subset \mathbb{P}^{n}$ satisfies the assumptions of Conjecture 1.1 and that $\alpha(I) \geqslant 2$. Then there is a subset $W \subset Z$ with the following properties:

(i) $W$ is of cardinality $\left(\begin{array}{l}d \\ n\end{array}\right)$.

(ii) For the ideal $J$ of $W$ we have $\alpha\left(J^{(k)}\right)=\alpha\left(I^{(k)}\right)$ for $k=1, \ldots, n$.

(iii) If $W$ is a star configuration, then $W=Z$.

Proof. To begin with, note that the equality in (10) together with Lemma 2.4 implies $\alpha\left(I^{(k)}\right)=d-n+k$ for $k=1, \ldots, n$.

The assumption $\alpha(I) \geqslant 2$ implies $d \geqslant n+1$. Since there is no form of degree $\leqslant d-n$ vanishing along $Z$, there must be at least

$$
s:=\left(\begin{array}{l}
d \\
n
\end{array}\right)
$$

points in $Z$.

We choose now exactly $s$ points $P_{1}, \ldots, P_{s}$ from $Z$ that impose independent conditions on forms of degree $d-n$. (This can be done, since vanishing at each point in $Z$ gives a linear equation on the coefficients of a form of degree $d-n$, so that we obtain a system of $\# Z$ linear equations of rank $s=\left(\begin{array}{l}d \\ n\end{array}\right)$ (which is the maximal possible rank). We choose then a subsystem of $s$ equations with maximal rank.) Let $W:=\left\{P_{1}, \ldots, P_{s}\right\}$ and let $J$ be the radical ideal of $W$. Since $W \subset Z$, we certainly have

$$
\alpha\left(J^{(k)}\right) \leqslant \alpha\left(I^{(k)}\right)
$$

for all $k \geqslant 0$. On the other hand we have

$$
\alpha(J)=d-n+1=\alpha(I)
$$

by the selection of $W$. Lemma 2.4 implies then that in fact

$$
\alpha\left(J^{(k)}\right)=\alpha\left(I^{(k)}\right)
$$

for $k=1, \ldots, n$. This shows that conditions (i) and (ii) are satisfied.

As for (iii): Suppose that $W$ is a star configuration. By (ii) we have $\alpha\left(I^{(n)}\right)=$ $\alpha\left(J^{(n)}\right)$, hence it follows from Lemma 4.1 that $W=Z$. 
Further, we will need the following elementary lemma on hypersurfaces that are obtained by taking derivatives.

Lemma 4.3. Let $X \subset \mathbb{P}^{n}$ be a hypersurface defined by a polynomial $f$ of degree $d$ with a point $P$ of multiplicity $m$ such that $2 \leqslant m<d$. Then there exists a direction $v$ such that the hypersurface $\Lambda$ defined by the directional derivative of $f$ in direction $v$ has multiplicity $m$ at $P$.

Proof. After a projective change of coordinates we may assume $P=(1: 0: \ldots: 0)$. Then we can write

$$
\begin{aligned}
f\left(x_{0}: x_{1}: \ldots: x_{n}\right)= & x_{0}^{d-m} g_{m}\left(x_{1}: \ldots: x_{n}\right) \\
& +x_{0}^{d-m-1} g_{m+1}\left(x_{1}: \ldots: x_{n}\right) \\
& +\ldots \\
& +g_{d}\left(x_{1}: \ldots: x_{n}\right)
\end{aligned}
$$

with homogeneous polynomials $g_{i}$ of degree $i$ for $i=m, \ldots, d$. Since $d>m$, the divisor defined by $\frac{\partial f}{\partial x_{0}}=0$ has multiplicity $m$ at $P$.

\section{Dimension 3}

In this section we give the

Proof of Theorem 1.2. We proceed by induction on $d$. For $d \leqslant 3$ the statement of the theorem is trivially satisfied, so we assume $d \geqslant 4$ now. By Lemma 4.2 we may assume the $Z$ is of cardinality $\left(\begin{array}{l}d \\ 3\end{array}\right)$. Let $X_{3} \subset \mathbb{P}^{3}$ be the divisor defined by a polynomial of degree $d$ in $I^{(3)}$. We assert that

$$
X_{3} \text { is reducible. }
$$

To see this, we first note that thanks to $m=3<4 \leqslant d$ there is by Lemma 4.3 for any $P \in Z$ a directional derivative surface $\Lambda_{P}$ of degree $d-1$ with multiplicity at least 3 at $P$. Arguing by contradiction, we assume that $X_{3}$ is irreducible, which implies that $X_{3}$ and $\Lambda_{P}$ intersect properly, i.e., in a curve. Adapting the proof of [11. Proposition 3.1] to dimension 3, we see that the linear system of forms of degree $d-2$ vanishing along $Z$ has only $Z$ as its base locus. (This is due to the fact that the regularity of $I$ is $d-2$.) We can therefore choose an element $Y$ in this system that does not contain any component of the intersection curve of $X_{3}$ and $\Lambda_{P}$. Then the three surfaces $X_{3}, \Lambda_{P}$ and $Y$ intersect in points only, and we can apply Bézout's theorem to get

$$
d(d-1)(d-2)=X \cdot \Lambda_{P} \cdot Y \geqslant 6\left(\left(\begin{array}{l}
d \\
3
\end{array}\right)-1\right)+9 .
$$

But this implies $0 \geqslant 3$, a contradiction. So $(*)$ is established.

Let now $\Gamma$ be an irreducible component of $X_{3}$ of smallest degree. Set $\gamma=\operatorname{deg} \Gamma$ and $X_{3}^{\prime}=X_{3}-\Gamma$. Our aim is to apply induction on $X_{3}^{\prime}$. To this end we consider the set

$$
Z^{\prime}=Z \backslash \Gamma
$$


It is non-empty, as otherwise $Z$ would be contained in $\Gamma$ and then $\alpha(I)$ would be less than $d-2$ : Indeed, we would have $\alpha(I) \leqslant \gamma \leqslant\left\lfloor\frac{d}{2}\right\rfloor$, which is less than $d-2$ if $d \geqslant 5$; and if $d=4$, then $\gamma=2$, so $X_{3}$ consists of two quadric, which implies that it can have only two triple points - but then $\alpha(I)=1$.

As $Z^{\prime}$ is non-empty, there is in particular a triple point on $X_{3}^{\prime}$, and hence $d-\gamma=$ $\operatorname{deg} X_{3}^{\prime} \geqslant 3$.

We claim that

$$
\alpha\left(I_{Z^{\prime}}\right) \geqslant d-\gamma-2 .
$$

In fact, there is otherwise a surface $S$ of degree $d-\gamma-3$ passing through $Z^{\prime}$, and then $S+\Gamma$ is a divisor of degree $d-3$ passing through $Z$, which contradicts the assumption $\alpha(I)=d-2$.

Next, note that

$$
\alpha\left(I_{Z^{\prime}}^{(3)}\right) \leqslant \operatorname{deg} X_{3}^{\prime}=d-\gamma .
$$

In fact, as $\Gamma$ does not pass through any of the points of $Z^{\prime}$, we know that $X_{3}^{\prime}$ has multiplicity at least three on $Z^{\prime}$.

By Lemma 2.4, we obtain from (5) the inequality

$$
\alpha\left(I_{Z^{\prime}}\right) \leqslant d-\gamma-2
$$

and this shows with (4) that equality holds in (6). From (5) we see then, again with Lemma 2.4. that equality holds in (5) as well. We have thus established that the assumptions of the theorem are satisfied for the set $Z^{\prime}$. By induction we conclude therefore that $Z^{\prime}$ is a star configuration and that $X_{3}^{\prime}$ decomposes into planes or $Z^{\prime}$ is contained in a hyperplane and then the support of $X_{3}^{\prime}$ is that hyperplane. As $\Gamma$ was chosen of minimal degree, it must therefore be a plane as well, and hence $X_{3}$ decomposes entirely into planes. We can then run the above induction argument for any plane component $\Pi$ of $X_{3}$ to see that the surface $X_{3}-\Pi$ yields a star configuration. This shows immediately that there are no triple intersection lines among these planes and we conclude by just counting points with multiplicity at least 3 (in fact exactly 3 ) that $Z$ is a star configuration.

\section{$6 \quad$ Further results and examples}

Recall that the Waldschmidt constant of a homogeneous ideal $I \subset \mathbb{P}^{n}$ is the asymptotic counterpart of the initial degree, defined as

$$
\widehat{\alpha}(I)=\lim _{k \rightarrow \infty} \frac{\alpha\left(I^{(k)}\right)}{k}=\inf _{k \geqslant 1} \frac{\alpha\left(I^{(k)}\right)}{k} .
$$

This invariant is indeed well defined, see [4] for this fact and some basic properties of $\widehat{\alpha}$. The Waldschmidt constants are interesting invariants that were recently rediscovered and studied by Bocci and Harbourne, see e.g. [5]. While Harbourne introduced the notation $\gamma(I)$, we propose here the notation $\widehat{\alpha}(I)$, as the Waldschmidt constant is the asymptotic version of the initial degree $\alpha(I)$, and the notation is then consistent with [9].

We state now a corollary of Theorem 1.2 dealing with the case when there is just one more $\alpha$-jump by 1 . 
Corollary 6.1. Let $Z$ be a finite set of points in projective three-space $\mathbb{P}^{3}$ and let $I$ be the radical ideal defining $Z$. If

$$
d:=\alpha\left(I^{(4)}\right)=\alpha(I)+3,
$$

then $\alpha(I)=1$, i.e., $Z$ is contained in a single plane in $\mathbb{P}^{3}$.

Proof. Using Theorem 1.2 we need to exclude the possibility that $Z$ forms a star configuration. To this end we apply in the case $n=3$ the inequality

$$
\widehat{\alpha}(I) \geqslant \frac{\alpha(I)+n-1}{n},
$$

which was proved by Demailly [6, Proposition 6]. The assumptions of his result are satisfied by our Theorem 1.2. Combining (8) with the fact that $\widehat{\alpha}(I) \leqslant \frac{\alpha\left(I^{(n+1)}\right)}{n+1}$ yields in our situation

$$
\frac{\alpha(I)+n-1}{n} \leqslant \frac{\alpha(I)+n}{n+1},
$$

which gives immediately $\alpha(I)=1$.

Remark 6.2 (Waldschmidt constant of star configuration). Note that there is equality in (8) for star configurations of points by [4, Proof of Theorem 2.4.3].

Remark 6.3. If Conjecture 1.1 holds for any $n$, then the proof of the above corollary shows that if $\alpha\left(I^{(n+1)}\right)=\alpha(I)+n$, then $\alpha(I)=1$.

We next provide examples showing that a single $\alpha$-jump by 1 is not sufficient in order to get the conclusion of Theorem 1.2 .

Example 6.4 (Kummer surface). In this example we show that in general the assumption

$$
\alpha\left(I^{(2)}\right)=\alpha(I)+1
$$

for an ideal $I$ of a set of points $Z$ in $\mathbb{P}^{3}$ is not sufficient in order to conclude that the points in $Z$ are coplanar or form a star configuration.

To this end, let $X \subset \mathbb{P}^{3}$ be the classical Kummer surface associated with an irreducible principally polarized abelian surface, and let $Z$ be the set of the 16 double points on $X$. It is well known (see e.g. [2, Sect. 10.2]) that these 16 points form a $16_{6}$ configuration, i.e., there are 16 planes $\Pi_{i}$ in $\mathbb{P}^{3}$ such that each plane $\Pi_{i}$ contains exactly 6 double points of $X$ (and exactly 6 planes pass through every point in $Z$ ). We claim that

$$
\alpha(I)=3 \quad \text { and } \quad \alpha\left(I^{(2)}\right)=4,
$$

where $I$ is the radical ideal of $Z$.

Granting this for a moment, we see immediately that the points in $Z$ are neither coplanar nor form a star configuration, whereas the assumption in (9) is satisfied.

Turning to the proof, assume that there exists a surface $S$ defined by an element of degree 3 in $I^{(3)}$. Let $\Pi$ be one of the 16 planes $\Pi_{i}$. Then

$$
1 \cdot 3 \cdot 4=\Pi \cdot S \cdot X \geqslant 6 \cdot 1 \cdot 2 \cdot 2
$$

implies that $\Pi$ is a component of $S$. As the same argument works for all 16 planes, we get a contradiction. Hence $\alpha\left(I^{(2)}\right)=4$.

A similar argument excludes the possibility that $Z$ is contained in a quadric. We leave the details to the reader. 
The following simpler example exhibiting the same phenomenon has been suggested by the referee.

Example 6.5. Let $L_{1}, L_{2}, L_{3}$ be mutually distinct and not coplanar lines in $\mathbb{P}^{3}$ intersecting in a point $P$. Let $A, B \in L_{1}, C, D \in L_{2}$ and $E \in L_{3}$ be points on these lines different from their intersection point $P$. Obviously the set $Z=\{A, B, C, D, E\}$ is not a star configuration. Let $I$ be the radical ideal of $Z$. Then it is elementary to check that

$$
\alpha(I)=2 \text { and } \alpha\left(I^{(2)}\right)=3 .
$$

Note that 5 is the minimal number of non-coplanar points that can give $\alpha\left(I^{(2)}\right)=$ $\alpha(I)+1$ and not form a star configuration.

The next example has been also suggested by the referee and replaces a much more complicated example of our original draft.

Example 6.6 (Five general points in $\mathbb{P}^{3}$ ). In this example we show that in general the assumption

$$
\alpha\left(I^{(3)}\right)=\alpha\left(I^{(2)}\right)+1
$$

for an ideal $I$ of a set of points $Z$ in $\mathbb{P}^{3}$ is also not sufficient in order to conclude that the points in $Z$ are coplanar or form a star configuration.

To this end let $Z=\{A, B, C, D, E\}$ consist of 5 points in general linear position in $\mathbb{P}^{3}$. For the radical ideal $I$ of $Z$ one has then

$$
\alpha\left(I^{(3)}\right)=\alpha\left(I^{(2)}\right)+1
$$

In fact, one has in this case

$$
\alpha(I)=2, \quad \alpha\left(I^{(2)}\right)=4 \text { and } \alpha\left(I^{(3)}\right)=5 .
$$

Acknowledgement. We would like to thank Jean-Pierre Demailly for bringing reference [6] to our attention. Further, we thank Brian Harbourne for helpful discussions. We thank also the referee for detailed helpful remarks, comments and nice examples for Section 6 .

\section{References}

[1] Baczyńska, M., Dumnicki, M., Habura, A., Malara, G., Pokora, P., Szemberg, T., Szpond, J., Tutaj-Gasińska, H.: Points fattening on $\mathbb{P}^{1} \times \mathbb{P}^{1}$ and symbolic powers of bi-homogeneous ideals. J. Pure Appl. Alg. 2013, http://www.sciencedirect.com/science/article/pii/S0022404913002491

[2] Birkenhake, C., Lange, H.: Complex Abelian Varieties. Berlin, Springer, 2010.

[3] Bocci, C., Chiantini, L.: The effect of points fattening on postulation, J. Pure Appl. Alg. 215 (2011), 89-98

[4] Bocci, C., Harbourne, B.: Comparing Powers and Symbolic Powers of Ideals, J. Algebraic Geometry 19 (2010), 399-417

[5] Bocci, C., Harbourne, B.: The resurgence of ideals of points and the containment problem. Proc. Amer. Math. Soc. 138 (2010), 1175-1190 
[6] Demailly, J.-P.: Formules de Jensen en plusieurs variables et applications arithmétiques, Bull. Soc. Math. France 110 (1982), 75-102

[7] Dumnicki, M., Szemberg, T., Tutaj-Gasińska, H.: Symbolic powers of planar point configurations. J. Pure Appl. Alg. 217 (2013), 1026-1036

[8] Eisenbud, D.: Commutative algebra. With a view toward algebraic geometry. Springer-Verlag, New York, 1995.

[9] de Fernex, T., Küronya, A., Lazarsfeld, R.: Higher cohomology of divisors on a projective variety. Math. Ann. 337 (2007), 443-455

[10] Geramita, A., Harbourne, B., Migliore, J.: Star configurations in $\mathbb{P}^{n}$. J. Algebra 376 (2013), 279-299

[11] Harbourne, B., Huneke, C.: Are symbolic powers highly evolved? J. Ramanujan Math. Soc. 28 (2013), 311-330

[12] Janssen, M.: On the fattening of lines in $\mathbb{P}^{3}$. arXiv:1306.4387

[13] Lazarsfeld, R.: Positivity in Algebraic Geometry. I.-II. Ergebnisse der Mathematik und ihrer Grenzgebiete, Vols. 48-49., Springer Verlag, Berlin, 2004.

[14] Sidman, J., Sullivant, S.: Prolongations and computational algebra. Canad. J. Math. 61 (2009), 930-949

Thomas Bauer, Fachbereich Mathematik und Informatik, Philipps-Universität Marburg, Hans-Meerwein-Straße, D-35032 Marburg, Germany

E-mail address: tbauer@mathematik.uni-marburg.de

Tomasz Szemberg, Instytut Matematyki UP, Podchorạżych 2, PL-30-084 Kraków, Poland

E-mail address: tomasz.szemberg@gmail.com 